\title{
Voltammetric sensor for D-penicillamine determination based on its electrocatalytic oxidation at the surface of ferrocenes modified carbon paste electrodes
}

\author{
JAHAN-BAKHSH RAOOF*, REZA OJANI and FERESHTEH CHEKIN \\ Electroanalytical Chemistry Research Laboratory, Department of Chemistry, \\ Faculty of Basic Science, Mazandaran University, Babolsar, Iran \\ e-mail: j.raoof@umz.ac.ir
}

MS received 20 December 2008; revised 20 April 2009; accepted 7 September 2009

\begin{abstract}
Electrocatalytic oxidation of D-penicillamine (D-PA) at the surface of ferrocene modified carbon paste electrode (FCCPE) was thoroughly investigated in aqueous solution with various $p \mathrm{H}$. The performance of this modified electrode was compared with those of 2,7-bis(ferrocenyl ethyl) fluoren-9one modified carbon paste electrode (2,7-BFEFMCPE). In the optimum condition, the oxidation of D-PA at the surface of FCCPE and 2,7-BFEFMCPE is occurred about 480 and $320 \mathrm{mV}$ less positive than that unmodified carbon paste electrode, respectively. The linear dynamic ranges $6 \times 10^{-5} \mathrm{M}-2 \times 10^{-3} \mathrm{M}$, $6.5 \times 10^{-5} \mathrm{M}-1.1 \times 10^{-3} \mathrm{M}$ and $7 \times 10^{-6} \mathrm{M}-1.6 \times 10^{-4} \mathrm{M}, 7 \times 10^{-6} \mathrm{M}-2 \times 10^{-4} \mathrm{M}$ of D-PA are obtained from CV and DPV methods for FCCPE and 2,7-BFEFMCPE, respectively. The detection limits $(3 \sigma)$ were determined as $5.4 \times 10^{-5} \mathrm{M}$ and $6.3 \times 10^{-5} \mathrm{M}$ in $\mathrm{CV}$ and $6.2 \times 10^{-6} \mathrm{M}$ and $6.8 \times 10^{-6} \mathrm{M}$ in DPV determinations for FCCPE and 2,7-BFEFMCPE, respectively. The proposed method was applied in a highly sensitive determination of D-PA in drug and human synthetic serum samples by standard addition and recovery methods, respectively.
\end{abstract}

Keywords. D-Penicillamine; ferrocenes; carbon paste electrode; electrocatalytic.

\section{Introduction}

D-Penicillamine, D-PA (2-amino-3-mercapto-3-methylbutanoic acid, 3-mercaptovaline and $\beta, \beta$-dimethylcysteine) is an unphysiological sulfurcontaining amino acid that belongs to the aminothiol family with a hydrogen atom in the beta-carbon of cysteine replaced by the methyl group. It can exist in $\mathrm{D}-$ and L-enantiomeric forms; however only the Dtype is clinically useful due to excessive toxicity of the L-type. ${ }^{1}$ This compound is derived from hydrolytic degradation of penicillin but it does not have antibiotic activity. ${ }^{2,3}$

D-Penicillamine is a thiol drug used in the treatment of Wilson's disease and an antosomal recessive disorder of copper transport. ${ }^{4}$ It is also used as antifibrotic agent to treat scleroderma ${ }^{5}$ and as antirheumatic drug to treat patients with active rheumatoid arthritis. ${ }^{6}$ D-penicillamine is rapidly absorbed from the gastrointestinal tract and is rapidly oxidized to various disulphide forms. ${ }^{7}$ Due to the biological importance of D-PA, its determination is interesting.

\footnotetext{
*For correspondence
}

There have been many attempts at automating the determination of penicillamine in pharmaceutical preparations by use of non-separation flow techniques $^{8}$ in conjunction with spectrophotometric, ${ }^{9}$ fluorimetric, ${ }^{10}$ electrochemical $^{11,12}$ and chemiluminescence-based ${ }^{13}$ detection. Among these methods, the electrochemical detection of thiols has been very successful for application in pharmaceutical and clinical preparations. ${ }^{14}$ The poor selectivity, slow kinetic of electrooxidation and very weak voltammetric responses of the target analyte at bare (unmodified) electrodes are often serious problems in detection with these electrodes. The chemical modification of electrodes using electron transfer mediators is an interesting field in this area. One of the most important effects of any mediator is a reduction of the overpotential required for electrochemical reaction and increase of the sensitivity and selectivity of method. ${ }^{15}$ Chemically modified electrodes based on reversible redox mediators such as ferrocenes, ${ }^{16-22}$ hydroquinones ${ }^{23}$ and catechols ${ }^{24,25}$ have been widely used for the electrocatalytic detection of some sulfur-containing amino acids. 
On the other hand, a practical mediator needs to have a low relative molar mass while being reversible, fast reacting, regenerated at low potential, $p \mathrm{H}$ independent, stable in both oxidized and reduced forms, unreactive with oxygen and non-toxic. Among the most successful mediators are those based on ferrocene and its derivatives that meet the above criteria. Ferrocenes have found broad applications as outer sphere redox mediators in solution and neutral redox-active component in carbon paste electrodes for the detection in aqueous solution because of their fast electron transfer rate, their broad range of $E^{0}$ that is adjusted by the substituents attached to the cyclopentadien ring system and the ease of their synthesis ${ }^{26}$. This work presents the study of the electrochemical behaviour of D-PA at surface of FCCPE and 2,7-BFEFMCPE. Detailed studies were performed using cyclic voltammetry, differential pulse voltammetry and double step potential chronoamperometry methods. The mechanism of the anodic oxidation of D-PA was elucidated and compared with those of 2,7-BFEFMCPE. These electrodes have been successfully applied for a highly sensitive determination of D-PA in pharmaceutical preparations and also for a recovery test of the drug spiked in phosphate buffer solution and human synthetic serum.

\section{Experimental}

\subsection{Chemicals}

The solvent used for the electrochemical studies was twice distilled water. Buffer solutions were prepared from orthophosphoric acid and its salts in the $p \mathrm{H}$ ranges from 2.00-10.00. High viscosity paraffin (density $=0.88 \mathrm{~g} \mathrm{~cm}^{-3}$ ) from Fluka was used as the pasting liquid for the carbon paste electrode. Graphite powder (particle diameter $=0.1 \mathrm{~mm}$ ) from Merck was used as the working electrode (WE) substrate. Potassium chloride from Fluka was used as the supporting electrolyte. The ferrocene and D-PA were from Fluka and were used as received. The 2,7bis(ferrocenyl ethyl) fluoren-9-one was (scheme 1) prepared by a reported procedure. ${ }^{27}$ All other reagents were of analytical grade.

\subsection{Apparatus}

The electrochemical experiments were performed using an Autolab Potentiostat/Galvanostat (Nether- lands) coupled with a Pentium IV personal computer with a standard three electrode configuration. A platinum wire was used as the auxiliary electrode. The FCCPE and 2,7-BFEFMCPE were used as the working electrode. The reference electrode was $\mathrm{Ag}|\mathrm{AgCl}| \mathrm{KCl}_{3 \mathrm{M}}$ (Metrohm). Also, a $p \mathrm{H}$-meter (Ion Analyzer 250, Corning) was used to read a $p \mathrm{H}$ of the buffered solution.

\subsection{Pharmaceutical preparations}

The D-PA formulations available were as D-PA capsules (containing $250 \mathrm{mg}$ D-PA, Laboratories Rubio, SA, Spain). Stock solutions of D-PA were freshly prepared as required in $0 \cdot 10 \mathrm{M}$ phosphate buffer at the desired $p \mathrm{H}(2 \cdot 00-10 \cdot 00)$ and protected from light during investigation. D-, L-alanine, L-arginine, Laspartic acid, glycine, L-histidine, L-lysine, D-, Lmethionine, glutathione, L-phenylalanine, L-serine, D-, L-tryptophan and L-cysteine for preparation of synthetic human serum were purchased from Merck and were of analytical reagent grade. In measurements, the composition of the synthetic serum was chosen near to its normal level in real human serum. $^{28}$

\subsection{Preparation of the modified electrode}

A $1 \%(w / w)$ ferrocene spiked carbon powder was made by dissolving the given quantity of ferrocene in diethyl ether and hand mixing with 99 times its weight of graphite powder with a mortar and pestle. The solvent was evaporated by stirring a mixture and paraffin was blended by hand-mixing and the resulting paste was inserted in the bottom of a glass tube (with internal radius $1.5 \mathrm{~mm}$ ). The electrical connection was implemented by a copper wire lead fitted into a glass tube. A carbon paste electrode without ferrocene was used as a blank to determine the background current. The 2,7-bis(ferrocenyl ethyl) fluoren-9-one modified carbon-paste elec-

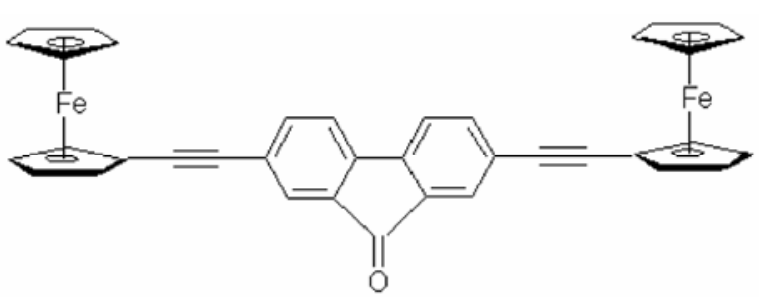

Scheme 1. 
trode was prepared by the same procedure as that of ferrocene by mixing of $(1 \% \mathrm{w} / \mathrm{w})$ of $2,7-b i s$ (ferrocenyl ethyl) fluoren-9-one with unmodified paste.

\section{Results and discussion}

\subsection{Electrochemical properties of FCCPE and 2,7-BFEFMCPE}

We have previously shown that a carbon paste electrode spiked with ferrocene and 2,7-bis(ferrocenyl ethyl) fluoren-9-one is constructed by the incorporation of ferrocene and 2,7-bis(ferrocenyl ethyl) fluoren-9-one in a graphite powder paraffin oil matrix. ${ }^{17,29}$ Their cyclic voltammograms (figure la and $b$ ) exhibit one anodic and cathodic peak corresponding with redox system $\mathrm{Fc} / \mathrm{Fc}^{+}$, which show a quasi-reversible behaviour in an aqueous medium. The cyclic voltammogram of bare CPE in pure supporting electrolyte shows no anodic or cathodic peaks (figure lc).

The effect of scan rates on the peak currents at the modified CPE in buffered phosphate solution $(0 \cdot 1 \mathrm{M}$, $p \mathrm{H} \mathrm{7.00)}$ were investigated by cyclic voltammetry (figure 2A). As shown in figure $2 \mathrm{~B}$, the anodic and cathodic peak current of FCCPE increases linearly with the square root of scan rate, which indicates diffusion controlled oxidation process occurring at the modified CPE. Also, the anodic and cathodic peak potential of modified electrode shift toward positive and negative potential, respectively. Therefore, the anodic and cathodic peak separation increases (figure 2A).

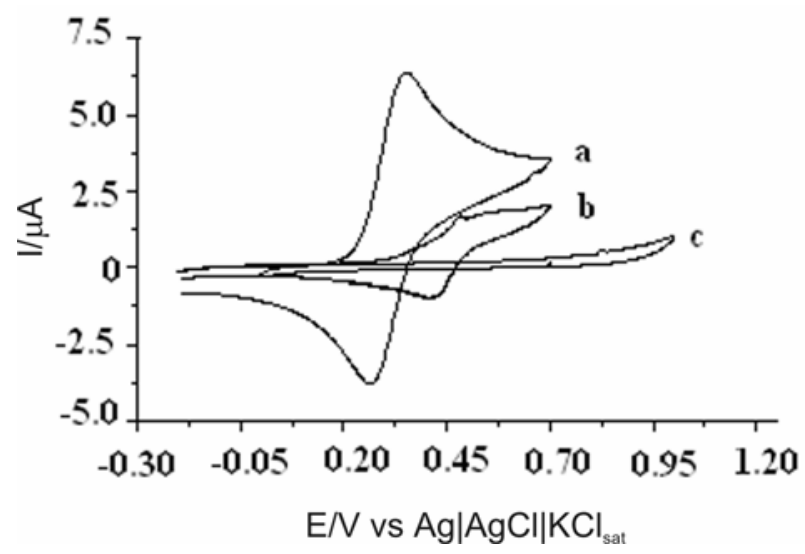

Figure 1. The cyclic voltammograms of (a) FCCPE, (b) 2,7-BFEFMCPE and (c) bare CPE in $0.1 \mathrm{M}$ phosphate buffer solution $(p \mathrm{H} \mathrm{7.00)}$ containing $\mathrm{KCl} 0.1 \mathrm{M}$ as supporting electrolyte at a scan rate $20 \mathrm{mV} \mathrm{s}^{-1}$.
The electrode capability for the generation of a reproducible surface was examined by cyclic voltammetric data obtained in optimum solution $p \mathrm{H}$ from five separately prepared these modified carbon paste electrodes (table 1). The calculated RSD for various parameters accepted as the criteria for a satisfactory surface reproducibility (about 1-6\%).

\section{2 pH optimization of the solution}

It well-known that the electrochemical behaviour of $\mathrm{D}-\mathrm{PA}$ is dependent on the $p \mathrm{H}$ value of the aqueous solution, ${ }^{15}$ whereas the electrochemical properties of
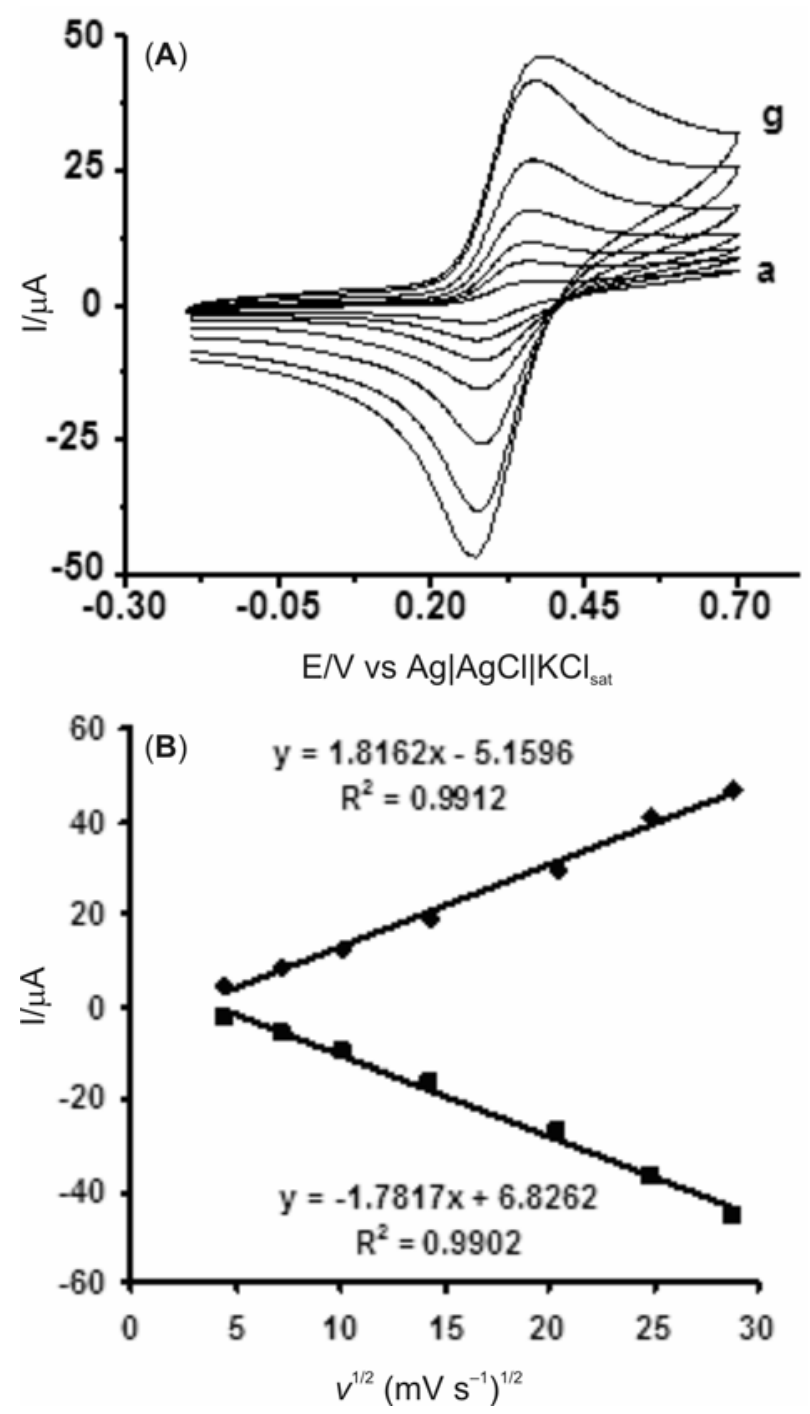

Figure 2. (A) Cyclic voltammograms of FCCPE in $0 \cdot 1 \mathrm{M}$ phosphate buffer solution $(p \mathrm{H} \mathrm{7.00)}$ at various scan rates: (a) 20, (b) 50, (c) 100, (d) 200, (e) 400, (f) 600 and (g) $800 \mathrm{mV} \mathrm{s}^{-1}$. (B) Plot of anodic and cathodic peak currents vs $v^{1 / 2}$ from cyclic voltammograms of (A). 
Table 1. Cyclic voltammetric data obtained for constructed FCCPE and 2,7-BFEFMCPE in $0 \cdot 1 \mathrm{M}$ phosphate buffer solution $(p \mathrm{H} 7 \cdot 00)$ at $20 \mathrm{mV} \mathrm{s}^{-1}$.

\begin{tabular}{lcccccc}
\hline Electrode & $E_{\mathrm{pa}}(\mathrm{V})^{\mathrm{a}}$ & $E_{\mathrm{pc}}(\mathrm{V})^{\mathrm{a}}$ & $E_{1 / 2}(\mathrm{~V})^{\mathrm{a}}$ & $\Delta E p(\mathrm{~V})^{\mathrm{a}}$ & $I_{\mathrm{pa}}(\mu \mathrm{A})$ & $I_{\mathrm{pc}}(\mu \mathrm{A})$ \\
\hline FCCPE & $0 \cdot 35[4 \cdot 8]$ & $0.27[1 \cdot 3]$ & $0 \cdot 31[0 \cdot 9]$ & $0.08[1 \cdot 3]$ & $6 \cdot 37[3 \cdot 7]$ & $3.78[4 \cdot 2]$ \\
2,7-BFEFMCPE & $0.49[3 \cdot 4]$ & $0 \cdot 41[1.4]$ & $0.45[0 \cdot 9]$ & $0.08[2 \cdot 8]$ & $2 \cdot 81[5 \cdot 7]$ & $1.61[2 \cdot 9]$ \\
\hline
\end{tabular}

${ }^{\mathrm{a}}$ Versus $\mathrm{Ag}|\mathrm{AgCl}| \mathrm{KCl}_{3 \mathrm{M}}$ as reference electrode

${ }^{\mathrm{b}}$ The values in parentheses indicate the calculated RSD (\%)
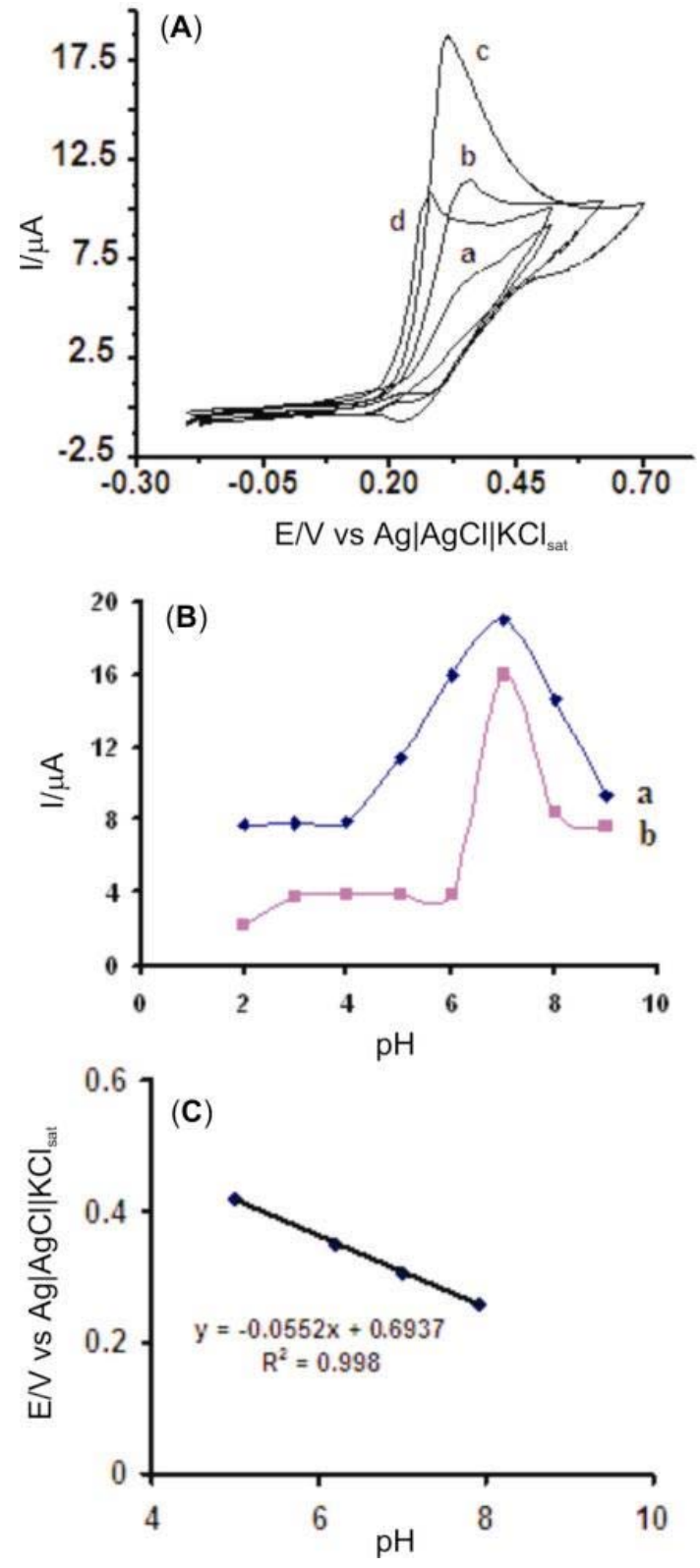

Figure 3. (A) Cyclic voltammograms of FCCPE in the presence of $1.0 \mathrm{mM}$ D-PA in $0.1 \mathrm{M}$ phosphate buffer solution with various $p \mathrm{H}$ values: (a) 5.00, (b) 6.00, (c) $7 \cdot 00$ and (d) 8.00 at scan rate $20 \mathrm{mV} \mathrm{s}^{-1}$. (B) The variation curve of the anodic peak current vs $p \mathrm{H}$ values at the surface of (a) FCCPE and (b) 2,7-BFEFMCP. (C) Variation of the electrocatalytic oxidation peak potential of D-PA at the FCCPE vs $p \mathrm{H}$ values (from CVs of (A)).
$\mathrm{Fc} / \mathrm{Fc}^{+}$redox couple of mediators are independent of the $p \mathrm{H}^{17}$ Therefore, $p \mathrm{H}$ optimization of the solution seems to be necessary in order to obtain the electrocatalytic oxidation of D-PA. Thus, we studied the electrochemical behaviour of D-PA in $0.1 \mathrm{M}$ phosphate buffered solutions with various $p \mathrm{H}$ values $(2.00<p \mathrm{H}<10.00)$ at the surface of FCCPE and 2,7-BFEFMCPE by cyclic voltammetry (figure $3 \mathrm{~A}$ ). It was found that the electrocatalytic oxidation of DPA at the surface of FCCPE and 2,7-BFEFMCPE was more favoured under neutral condition than in an acidic medium. This appeared as a gradual growth in the anodic peak current and a simultaneous decrease in the cathodic peak current of cyclic voltammograms. As can be seen in figure 3B, electrocatalytic current for electrooxidation of D-PA is high at a biological $p \mathrm{H}(p \mathrm{H} \mathrm{7.00)}$. Therefore, $p \mathrm{H} \mathrm{7.00}$ was chosen as the optimum $p \mathrm{H}$ for electrocatalysis of D-PA at surface of FCCPE and 2,7-BFEFMCPE. The potential- $p \mathrm{H}$ diagram was constructed by plotting the oxidation peak potential values of D-PA as a function of $p \mathrm{H}$ (figure 3C). This diagram comprises a linear segment with slope value equal to $55 \mathrm{mV}$ per unit of $p \mathrm{H}$ (a two-proton and two-electron process). According to the slope value, the reaction scheme would probably via the following mechanistic steps, which protonated and deprotonated forms of D-penicillamine (RSH in below) can be oxidized by $\mathrm{Fc}^{+}$produced in the surface of electrode:

$$
\begin{aligned}
& 2 F c \rightarrow 2 \mathrm{Fc}^{+}+2 e^{-}, \\
& 2\left(\mathrm{RSH}^{\circ} \mathrm{RS}^{-}\right)+2 \mathrm{Fc}^{+} \rightarrow \mathrm{RS}-\mathrm{SR}+2 \mathrm{Fc}\left(+2 \mathrm{H}^{+}\right) .
\end{aligned}
$$

\subsection{Mediated oxidation of D-PA}

A comparison of the cyclic voltammograms of FCCPE and 2,7-BFEFMCPE in buffered phosphate solution $(0.1 \mathrm{M}, p \mathrm{H} \mathrm{7.00)}$ in the absence (figure 1) and presence of D-PA (figure 4), demonstrates that the electrooxidation of D-PA can be catalysed by the ferrocinium produced from electrooxidation of 
ferrocene and 2,7-bis(ferrocenyl ethyl) fluoren-9one, incorporated in the carbon paste electrode. The results show that, at the bare CPE, D-PA oxidation occurs irreversibly with a peak potential of nearly $800 \mathrm{mV}$ versus $\mathrm{Ag}|\mathrm{AgCl}| \mathrm{KCl}_{3 \mathrm{M}}$ (figure $4 \mathrm{a}$ ), whereas in the absence of D-PA no peaks appears (figure 1c). In the presence of D-PA $\left(10^{-3} \mathrm{M}\right)$, the anodic peak currents that are observed for FCCPE and 2,7BFEFMCPE increase greatly, while the corresponding cathodic peaks disappear on the reverse scan (figure $4 \mathrm{~b}$ and $\mathrm{c}$ ). Therefore the oxidation of D-PA at surface of FCCPE and 2,7-BFEFMCPE occurs at a potential about 480 and $320 \mathrm{mV}$, respectively which is less positive than at bare carbon paste electrode.

A significant increase of the anodic peak current in conjunction with the sharpness of the peak, which is related to a reduction of the overpotential of the process at the surface of the modified electrode, revealed that the modified electrode could act as a very effective promoter to enhance the kinetics of the electrochemical process. By using ferrocene and

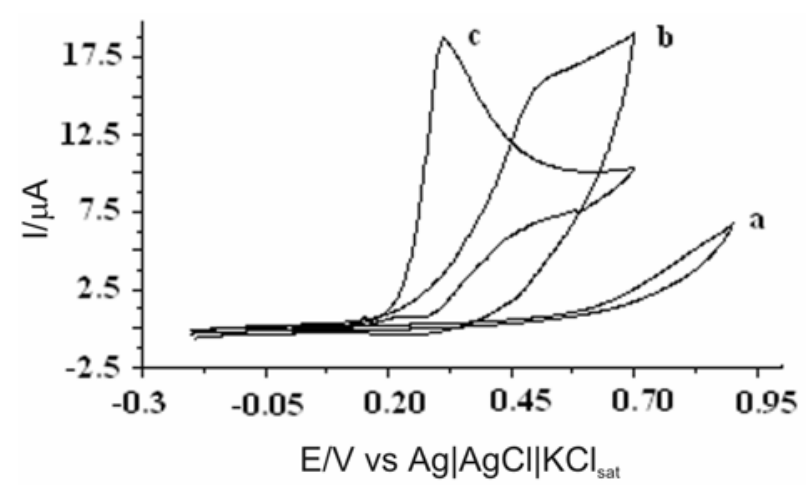

Figure 4. Cyclic voltammograms of (a) CPE, (b) 2,7BFEFMCP and (c) FCCPE in the presence of $1 \mathrm{mM}$ D-PA at scan rate $20 \mathrm{mV} \mathrm{s}^{-1}$ in $0.1 \mathrm{M}$ phosphate buffer

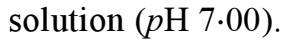

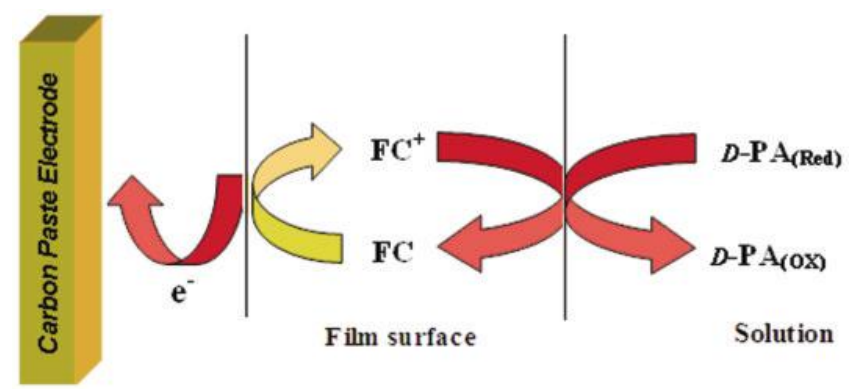

Scheme 2. 2,7-bis(ferrocenyl ethyl) fluoren-9-one as an electron mediator in the matrix of the modified electrode, the overpotential for the anodic oxidation of D-PA becomes considerably lower and then rate of the heterogeneous electron transfer is increased with the $\mathrm{EC}^{\prime}$ mechanism proposed in scheme 2. A comparison between the electro-oxidation of D-PA at the surface of two different modified electrodes (FCCPE and 2,7-BFEFMCPE) is shown in figure 4. The peak potential for the FCCPE electrode occurred at about $320 \mathrm{mV}$ vs $\mathrm{Ag}|\mathrm{AgCl}| \mathrm{KCl}_{3 \mathrm{M}}$ in buffered $p \mathrm{H}$ 7.00 solution at a scan rate of $20 \mathrm{mV} \mathrm{s}^{-1}$, which is nearly $160 \mathrm{mV}$ less positive than that for the 2,7BFEFMCPE. The results show that FCCPE can be considerably more effective in decreasing the overpotential of anodic oxidation of D-PA, a very sharp anodic wave being obtained at lower positive potentials. The electrocatalytic oxidation of D-PA has been investigated at the surface of other chemically modified electrodes. As shown, the shifted potential of D-PA electrooxidation at surface of FCCPE is bigger than other electrodes. The linear dynamic rang (LOR) and limit of detection (LOD) of FCCPE is comparable with other results (see table 2). $15,24,31,32$

The values of $\alpha n_{a}$ (where $\alpha$ is the transfer coefficient and $n_{a}$ is the number of electrons involved in the rate determining step) were calculated for the oxidation of D-PA at $\mathrm{pH} 7.00$ at both the modified and unmodified carbon paste electrodes, according to the equation: ${ }^{33}$

$$
\alpha n_{\mathrm{a}}=0 \cdot 048 /\left(E_{p}-E_{p / 2}\right),
$$

where $E_{p / 2}$ is the potential corresponding to $I_{p / 2}$. The values for $\alpha n_{\mathrm{a}}$ were found to be $0.42,0.37$ and 0.27 for the oxidation of D-PA at surface of the FCCPE, 2,7-BFEFMCPE and CPE, respectively. These values clearly show that not only the overpotential for the D-PA oxidation is reduced at the surface of the FCCPE, but also the rate of the electron transfer process is greatly enhanced; this phenomenon is confirmed by the larger $I_{p a}$ values recorded during cyclic voltammetry at the FCCPE.

\subsection{Chronoamperometry}

Double potential step chronoamperometry, as well as other electrochemical methods were employed for the investigation of electrode processes at FCCPE and 2,7-BFEFMCPE. Figure 5A shows the current-time curves of FCCPE obtained by setting the working 
Table 2. Comparison of the efficiency of some modified electrodes in the electrocatalytic oxidation of D-PA.

\begin{tabular}{llccccc}
\hline Electrode & \multicolumn{1}{c}{ Modifier } & $p \mathrm{H}$ & $\begin{array}{c}\text { Peak potential } \\
\text { shift }(\mathrm{mV})\end{array}$ & LDR $(\mu \mathrm{M})$ & LOD $(\mu \mathrm{M})$ & References \\
\hline CP & Cobalt salophen & $3 \cdot 00$ & 50 & $10-1000^{\mathrm{a}}$ & $1 \cdot 00^{\mathrm{a}}$ & 15 \\
$\mathrm{GC}^{\mathrm{c}}$ & 4-tert-Butylcatechol & $7 \cdot 00$ & 420 & $0 \cdot 02-80^{\mathrm{b}}$ & $0 \cdot 007^{\mathrm{b}}$ & 24 \\
$\mathrm{CP}$ & Ferrocene carboxylic acid $^{\mathrm{b}}$ & $7 \cdot 00$ & 420 & $6 \cdot 5-100^{\mathrm{a}}$ & $6 \cdot 15^{\mathrm{a}}$ & 31 \\
$\mathrm{CP}$ & 1,4-FEPE & $7 \cdot 00$ & 300 & $7-230^{\mathrm{a}}$ & $2 \cdot 9^{\mathrm{a}}$ & 32 \\
$\mathrm{CP}$ & Ferrocene $_{\mathrm{CP}}$ & $7 \cdot 00$ & 480 & $7-160^{\mathrm{a}}$ & $6 \cdot 2^{\mathrm{a}}$ & This work \\
\hline
\end{tabular}

${ }^{a}$ LDR and LOD were obtained using differential pulse voltammetry

${ }^{\mathrm{b}} \mathrm{LDR}$ and LOD were obtained using cyclic voltammetry

${ }^{\mathrm{c}} \mathrm{GC}$ is on the top of the rotating biosensor

${ }^{\mathrm{d}} 1$-(4-(Ferrocenyl-ethynyl) phenyl]-1-ethanone

e 2,7-bis(ferrocenyl ethyl) fluoren-9-one

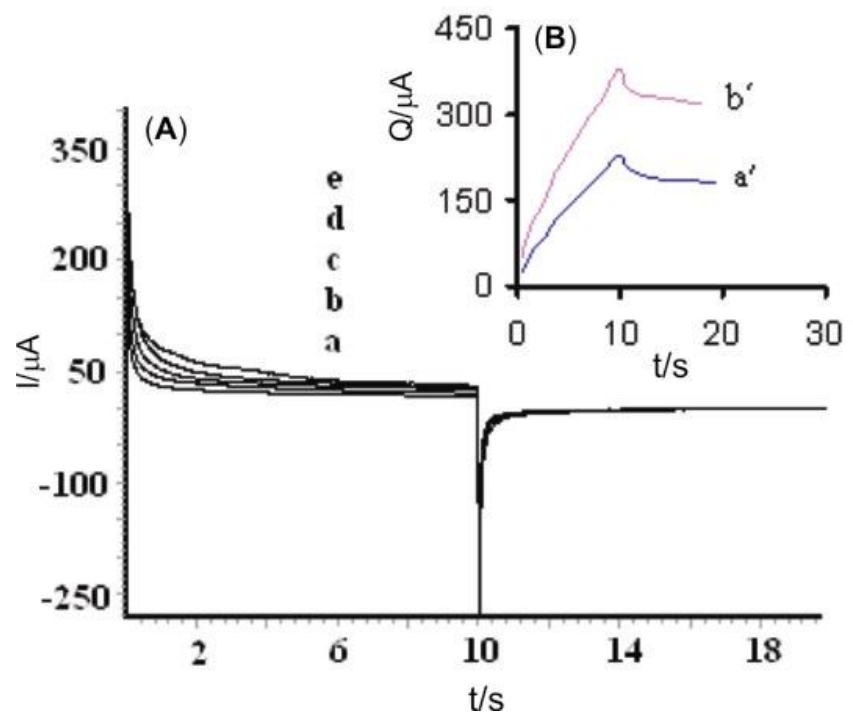

Figure 5. (A) Double step potential chronoamperograms obtained at the FCCPE in the (a) absence and presence of (b) $0 \cdot 5$, (c) $1 \cdot 0$, (d) 1.5 and (e) $2.0 \mathrm{mM}$ of D-PA in $0.1 \mathrm{M}$ phosphate buffer solution $(p \mathrm{H} \mathrm{7 \cdot 00)}$. First and second potential steps were 0.70 and $0.25 \mathrm{~V}$ vs $\mathrm{Ag}|\mathrm{AgCl}|$ $\mathrm{KCl}_{3 \mathrm{M}}$. (B) The charge-time curves: (a') for curve (a) and $\left(b^{\prime}\right)$ for curve (b) of chronoamperograms (A).

electrode potential at the $0.7 \mathrm{~V}$ (first potential step) and $0.25 \mathrm{~V}$ (second potential step) vs $\mathrm{Ag}|\mathrm{AgCl}| \mathrm{KCl}_{3 \mathrm{M}}$ for various concentrations of D-PA in buffered

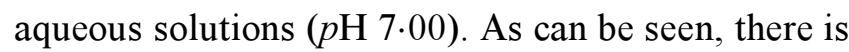
no net cathodic current corresponding to the reduction of $\mathrm{FC}^{+}$to $\mathrm{Fc}$ in the presence of D-PA, when the second potential step is employed. The oxidation current value increases with increasing of the D-PA concentration at first potential step. While the forward and backward potential step chronoamperometry on the modified electrode in the blank buffered solution show very symmetrical chronoamperograms with an equal charge consumed for the oxidation and reduction of $\mathrm{Fc} / \mathrm{Fc}^{+}$redox system in the CPE (figure $5 \mathrm{~B}$, curve $\mathrm{a}^{\prime}$ ). However, in the presence of D-PA the charge value associated with forward chronoamperometry is significantly greater than that observed for backward chronoamperometry (figure $5 \mathrm{~B}$, curve $\mathrm{b}^{\prime}$ ). A similar behaviour was observed for 2,7-BFEFMCPE.

For an electroactive material with a difficient (D), the current corresponding to the electrochemical reaction (under diffusion controlled) is described by Cotrell's equation: ${ }^{34}$

$$
I=n F A_{\mathrm{g}} D_{\mathrm{app}}{ }^{1 / 2} C_{0} \pi^{1 / 2} t^{-1 / 2},
$$

where $A_{\mathrm{g}}, D_{\text {app }}$ and $C_{0}$ are geometric area of the electrode (the diameter (d) of the FCCPE and 2,7BFEFMCPE were measured and then the geometric area of the them were calculated according to $\left.(\pi(d / 2) 2), 0.070 \mathrm{~cm}^{2}\right)$, the apparent diffusion coefficient $\left(\mathrm{cm}^{2} \mathrm{~s}^{-1}\right)$ and the bulk concentration $\left(\mathrm{mol} \mathrm{cm}^{-3}\right)$, respectively. The plot of I versus $t^{-1 / 2}$ would be linear, and from its slope, the mean value of the $D_{\text {app }}$ of D-PA was found to be $8.7 \times 10^{-6}$ and $7.4 \times$ $10^{-6} \mathrm{~cm}^{2} \mathrm{~s}^{-1}$ for FCCPE and 2,7-BFEFMCPE, respectively.

\subsection{Evaluation of catalytic reaction rate constant}

The rate constant for the electrochemical reaction can be evaluated by chronoamprerometry according to the method described in: ${ }^{35}$

$$
I_{\mathrm{C}} / I_{\mathrm{L}}=\pi^{1 / 2} \gamma^{1 / 2}=\pi^{1 / 2}\left(k_{h} C_{b} t\right)^{1 / 2},
$$


where $I_{C}$ is the current in the presence of D-PA and $I_{L}$ is the limited current in the absence of D-PA. The $k_{h}$ and $t$ are the electrochemical rate constant $\left(\mathrm{cm}^{3} \mathrm{~mol}^{-1} \mathrm{~s}^{-1}\right)$ and time elapsed (s). The above equation can be used to calculate the rate constant of the process, $k_{h}$. From the slope of $I_{C} / I_{L}$ versus $t^{1 / 2}$ plot, the value of $k_{h}$ can be simply calculated for a given concentration of substrate. From this plot, $k_{h}$ equal to $7.0 \times 10^{2}$ and $5.5 \times 10^{2} \mathrm{M}^{-1} \mathrm{~s}^{-1}$ for FCCPE and 2,7-BFEFMCPE, respectively was obtained.

\subsection{Linearity and sensitivity}

The electrocatalytic peak current of D-PA oxidation at the surface of FCCPE and 2,7-BFEFMCPE was used for determination of D-PA in solution. Figures 6 and 7 show the cyclic and differential pulse voltammograms that were performed using FCCPE in phosphate buffer solution containing various concentrations of D-PA. The results show the electrocatalytic peak current of D-PA oxidation at the surface of FCCPE and 2,7-BFEFMCPE was linearly dependent on the D-PA concentration and the range of this linearity depends on the amount of mediator in the electrode matrix. The mediated oxidation peak

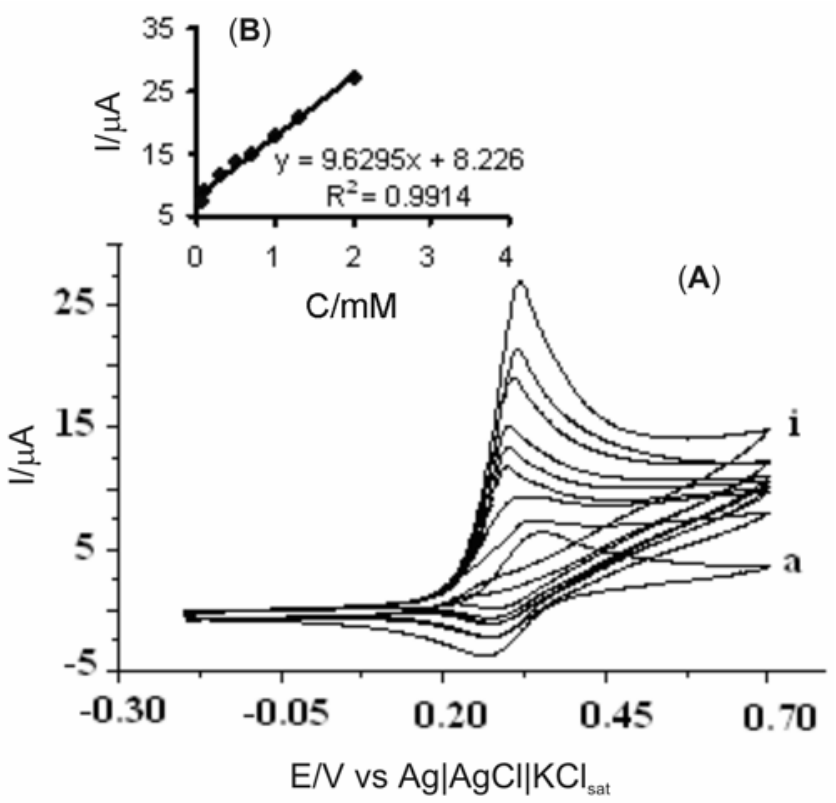

Figure 6. (A) Cyclic voltammograms of D-PA at various concentrations: (a) absence and (b) presence of 0.06 , (c) $0 \cdot 1$, (d) $0 \cdot 3$, (e) $0 \cdot 5$, (f) $0 \cdot 7$, (g) $1 \cdot 0$, (h) $1 \cdot 3$, and (i) $2.0 \mathrm{mM}$ at the surface of FCCPE in $0.1 \mathrm{M}$ phosphate buffer solution $\left(p \mathrm{H} \mathrm{7.00)}\right.$ at a scan rate of $20 \mathrm{mV} \mathrm{s}^{-1}$. (B) Plot of electrocatalytic peak currents (from CV of (A)) vs the D-PA concentrations. current of D-PA at the surface of a $1 \%$ FCCPE and 2,7-BFEFMCPE was proportional to the concentration of substrate. The linear dynamic ranges $6 \times$ $10^{-5} \mathrm{M}-2 \times 10^{-3} \mathrm{M}, 6.5 \times 10^{-5} \mathrm{M}-1.1 \times 10^{-3} \mathrm{M}$ and $7 \times 10^{-6} \mathrm{M}-1.6 \times 10^{-4} \mathrm{M}, \quad 7 \times 10^{-6} \mathrm{M}-2 \times 10^{-4} \mathrm{M}$ of D-PA are obtained with CV and DPV methods for FCCPE and 2,7-BFEFMCPE, respectively. The detection limits $(3 \sigma)$ were determined as $5.4 \times 10^{-5} \mathrm{M}$ and $6.3 \times 10^{-5} \mathrm{M}$ in $\mathrm{CV}$ and as $6.2 \times 10^{-6} \mathrm{M}$ and $6.8 \times 10^{-6} \mathrm{M}$ in DPV determinations for FCCPE and 2,7-BFEFMCPE, respectively. Thus, the catalytic oxidation of D-PA can readily be applied for the determination of D-PA.

The stability of the modified electrodes was investigated by measuring the current response of $1.0 \mathrm{mM}$ D-PA every few days. After 2 weeks, the response current of the modified electrodes decrease gradually to $80 \%$ of the initial value. The good stability of the modified electrodes offers interesting opportunity for practical applications in biosensing and bioengineering.

\subsection{Analytical application}

The proposed method for D-PA was applied to the determination of D-PA capsules (containing $250 \mathrm{mg}$ D-PA). The correlation coefficients of the calibration plots in the standard addition method were in the range of 0.9921-0.9946. The precision of the method was assessed by three repetitions of the analysis of

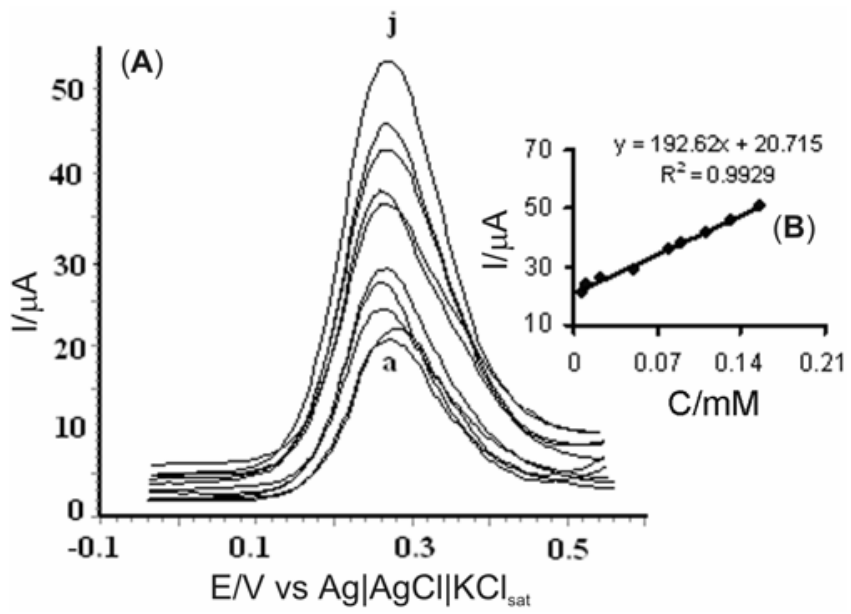

Figure 7. (A) Differential pulse voltammograms of D-PA at (a) absence and various concentrations: (b) presence of 0.007 , (c) 0.01 , (d) 0.03 , (e) 0.05 , (f) 0.08 , (g) $0 \cdot 10$, (h) $0 \cdot 11$, (i) $0 \cdot 13$ and (i) $0.16 \mathrm{mM}$ at the surface of FCCPE in $0 \cdot 1 \mathrm{M}$ phosphate buffer solution $(p \mathrm{H} 7 \cdot 00$. (B) Plot of electrocatalytic peak currents (from DPV of (A)) vs the D-PA concentrations. 
Table 3. Determination of tablet D-PA and recovery data obtained for D-PA added at specified concentrations to buffer solution and $(p \mathrm{H} 7 \cdot 00)$ and human synthetic serum.

\begin{tabular}{|c|c|c|c|c|c|c|}
\hline \multirow[b]{2}{*}{ Sample } & \multirow{2}{*}{$\begin{array}{l}\text { Labelled value } \\
\qquad(\mathrm{mg} / 10 \mathrm{ml})\end{array}$} & \multirow{2}{*}{$\begin{array}{l}\text { Amount found } \\
(\mathrm{mg} / 10 \mathrm{ml})\end{array}$} & \multicolumn{2}{|c|}{ Mean recovery $\pm \mathrm{SD}(\%)$} & \multirow[b]{2}{*}{$T_{\exp }$} & \multirow[b]{2}{*}{$F_{\exp }$} \\
\hline & & & Proposed-method $^{\mathrm{a}}$ & USP-method $^{\mathrm{b}}$ & & \\
\hline D-PA tablet & 1 & 0.99 & $99 \cdot 0 \pm 1 \cdot 9$ & $102 \pm 0 \cdot 6$ & $2 \cdot 36$ & $5 \cdot 68$ \\
\hline Sample $1+0 \cdot 1 \mathrm{mg}$ D-PA & - & $0 \cdot 10$ & $100 \pm 3 \cdot 4$ & & & \\
\hline Sample $1+0.5 \mathrm{mg}$ D-PA & - & $0 \cdot 47$ & $94 \cdot 0 \pm 4 \cdot 1$ & & & \\
\hline $10 \mathrm{ml} \mathrm{serum}+0.1 \mathrm{mg} \mathrm{D}-\mathrm{PA}$ & - & 0.097 & $97 \cdot 0 \pm 0.9$ & & & \\
\hline $10 \mathrm{ml} \mathrm{serum}+0.2 \mathrm{mg}$ D-PA & - & $0 \cdot 21$ & $105 \pm 1 \cdot 5$ & & & \\
\hline
\end{tabular}

${ }^{\mathrm{a}}$ Result based on three replicate determinations per samples

${ }^{\mathrm{b}}$ Result based on five replicate determinations per samples. Theoretical values for $t=2 \cdot 45$ and $F=6 \cdot 94(P=0 \cdot 05)$

the pharmaceutical preparations. Results of the recoveries of the D-PA added to $10.0 \mathrm{ml}$ of buffered $p \mathrm{H}$ 7.00 solutions and human synthetic serum are shown in table 3 . The excellent recovery results indicate that the constituents in the complex matrix of the serum sample do not interfere with the detection of D-PA and this voltammetric method can be used as a very highly sensitive detection device for D-PA in biological and pharmaceutical preparations.

\section{Conclusions}

The results of the present study show that ferrocene can be considerably more effective in lowering the overpotential of anodic oxidation of D-PA, a very sharp anodic wave obtained at lower positive potentials. The peak potential for FCCPE occurred at $320 \mathrm{mV}$ vs $\mathrm{Ag}|\mathrm{AgCl}| \mathrm{KCl}_{3 \mathrm{M}}$ in buffered $p \mathrm{H} \quad 7.00$ solution at a scan rate of $20 \mathrm{mV} \mathrm{s}^{-1}$, which is nearly $160 \mathrm{mV}$ less positive than that for the 2,7BFEFMCPE. The presence of ferrocene as an electron mediator, compared with an electrode modified with 2,7-bis (ferrocenyl ethyl) fluoren-9-one, decreases the anodic overpotential of D-PA considerably and enhances the anodic peaks in the CV and DPV methods significantly. Based on the voltammetric studies of electrooxidation of D-PA, a very highly sensitive method, DPV, is applied successfully for the determination of trace amounts of D-PA $(\mu \mathrm{M})$ in the clinical and pharmaceutical preparations. High sensitivity and a low detection limit, together with the very easy preparation and easy regeneration of the electrode surface and reproducibility makes the system discussed above useful in the construction of simple devices for the determination of D-PA.

\section{References}

1. Munro R and Capell H A 1997 Br. J. Rheumatol. 36 104

2. Walshe J M 2003 Mov. Disord. 18853

3. Chong C R and Auld D S 2000 Biochemistry 397580

4. Czlonkowska A, Gajda J and Rodo M 1996 J. Neurol. 243269

5. Leroy E C, Trojanowska M and Smith E A 1991 Clin. Exp. Rheumatol. 9173

6. Wangfuengkanagul $\mathrm{N}$ and Chailapakul O 2002 Talanta 581213

7. Kusmierek K and Bald E 2007 Anal. Chim. Acta 590 132

8. Suliman F E O, Al-Lawati H A J, Al-Kindy S M Z, Nour I E M and Salama S B A 2003 Talanta 61221

9. Garcia M S, Sanchez-pedreno C, Albero M I and Rodenas V 1993 J. Pharm. Biomed. Anal. 11633

10. Al-Majed A A 2000 Anal. Chim. Acta 408169

11. Catala Icardo M, Armenta Estrela O, Sajewicz M, Garcia Mateo J V and Martinez Calatayud J 2001 Anal. Chim. Acta 438281

12. Favaro G and Fiorani M 1996 Anal. Chim. Acta 332 249

13. Zhang Z D, Baeyens W R G, Zhang X R and Van der Weken G 1996 Analyst 1211569

14. Zhang S, Sun W, Zhang W, Qi W, Jin L, Yamamoto K, Tao S and Jin J 1999 Anal. Chim. Acta 38621

15. Shahrokhian S, Souri A and Khajehsharifi H $2006 J$. Electroanal. Chem. $\mathbf{5 6 5} 95$

16. Raoof J B, Ojani R and Kiani A $2001 \mathrm{~J}$. Electroanal. Chem. $\mathbf{5 1 5} 45$

17. Raoof J B, Ojani R and Karimi-Maleh 2007 Int. J. Electrochem. Sci. 2257

18. Raoof J B, Ojani R and Kolbadinezhad M 2005 Electroanalysis 172043

19. Raoof J B, Ojani R, Beitollahi H and Hossienzadeh R 2006 Anal. Sci. 221213

20. Suzuki $M$, Nakajima $R$, Tsuruta $M$, Higuchi $M$, Einaga Y and Yamamoto K 2006 Macromolecules 39 64

21. Khan M A K, Kerman K, Petryk M and Kraatz H B 2008 Anal. Chem. 802574 
22. Bu H Z, English A M and Mikkelsen S R 1996 Anal. Chem. 683951

23. Shahrokhian S and Bozorgzadeh S 2006 Electrochim. Acta 14271

24. Torriero A A J, Piola H D, Martinez N A, Panini N V, Raba J and Silber J J 2007 Talanta 71 1198

25. Torriero A A J, Salinas E, Marchevsky E J, Raba J and Silber J J 2006 Anal. Chim. Acta 580136

26. Asaftei S and Walder L 2004 Electrochim. Acta 49 4679

27. Hosseinzadeh R $1999 \mathrm{Ph}$ D thesis, Justus-LiebiyUniversitate, Giessen, Germany

28. Pau C P and Rechnitz G A 1984 Anal. Chim. Acta 160141
29. Raoof J B, Ojani R and Kolbadinezhad M 2005 Bull. Chem. Soc. Japan 78818

30. Tan W T, Bond A M, Ngooi S W, Lim E B and Goh J K 2003 Electrochim. Acta 491181

31. Raoof J B, Ojani R and Chekin F 2007 Electroanalysis 191883

32. Raoof J B, Ojani R, Chekin F and Hosseinzadeh R 2007 Int. J. Electochem. Sci. 2848

33. Takeuchi E S and Murray R W 1985 J. Electroanal. Chem. 18949

34. Greef R, Peter R, Pletcher D and Robinson J 1990 Instrumental methods in electrochemistry (Chichster: Ellis Horwood)

35. Galus Z 1976 Fundamentals of electrochemical analysis (New York: Ellis Hardwood) 